Journal of Integer Sequences, Vol. 9 (2006), Article 06.2.3

\title{
Evaluations of Some Variant Euler Sums
}

\author{
Hongwei Chen \\ Department of Mathematics \\ Christopher Newport University \\ Newport News, VA 23606 \\ hchen@cnu.edu
}

\begin{abstract}
In this note we present some elementary methods for the summation of certain Euler sums with terms involving $1+1 / 3+1 / 5+\cdots+1 /(2 k-1)$.
\end{abstract}

\section{Introduction}

In the last decade, based on extensive experimentation with computer algebraic systems, a large class of Euler sums have been explicitly evaluated in terms of the Riemann zeta function $\zeta(k)$. For example, let

$$
H_{k}=1+\frac{1}{2}+\cdots+\frac{1}{k}
$$

Then

$$
\begin{aligned}
\sum_{k=1}^{\infty} \frac{1}{k^{2}} H_{k} & =2 \zeta(3) \\
\sum_{k=1}^{\infty} \frac{1}{2^{k} k^{2}} H_{k} & =\zeta(3)-\frac{\pi^{2}}{12} \ln 2 \\
\sum_{k=1}^{\infty} \frac{1}{k^{2}} H_{k}^{2} & =\frac{17}{4} \zeta(4)
\end{aligned}
$$

and

$$
\sum_{k=1}^{\infty} \frac{(-1)^{k-1}}{k^{2}} H_{k}=\frac{5}{8} \zeta(3)
$$




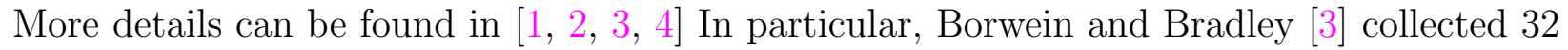
beautiful proofs of the first sum above.

Motivated by the above results, in this note, replacing $H_{k}$ by

$$
h_{k}=H_{2 k}-\frac{1}{2} H_{k}=1+\frac{1}{3}+\cdots+\frac{1}{2 k-1},
$$

we study the following variant Euler sums

$$
\sum_{k=1}^{\infty} a_{k} h_{k}
$$

where the $a_{k}$ are relatively simple function of $k$.

\section{The Main Results}

We begin to derive some series involving $h_{k}$. Since

$$
-\ln (1-x)=\int_{0}^{x} \frac{d t}{1-t}=\sum_{k=1}^{\infty} \frac{x^{k}}{k}
$$

replacing $x$ by $-x$ gives

$$
\ln (1+x)=\sum_{k=1}^{\infty} \frac{(-1)^{k-1} x^{k}}{k}
$$

Averaging these two series gives us

$$
\frac{1}{2} \ln \left(\frac{1+x}{1-x}\right)=\sum_{k=1}^{\infty} \frac{1}{2 k-1} x^{2 k-1} .
$$

In term of the Cauchy product and partial fractions, we have

$$
\begin{aligned}
\frac{1}{4} \ln ^{2}\left(\frac{1+x}{1-x}\right) & =\sum_{k=1}^{\infty}\left(\frac{1}{(2 k-1) \cdot 1}+\frac{1}{(2 k-3) \cdot 3}+\cdots+\frac{1}{1 \cdot(2 k-1)}\right) x^{2 k} \\
& =\sum_{k=1}^{\infty} \frac{1}{2 k}\left[\left(\frac{1}{2 k-1}+\frac{1}{1}\right)+\left(\frac{1}{2 k-3}+\frac{1}{3}\right)+\cdots+\left(\frac{1}{1}+\frac{1}{2 k-1}\right)\right] x^{2 k} \\
& =\sum_{k=1}^{\infty}\left(1+\frac{1}{3}+\cdots+\frac{1}{2 k-1}\right) \frac{x^{2 k}}{k} .
\end{aligned}
$$

Noting that $h_{k}$ is given by (1), we have

$$
\sum_{k=1}^{\infty} \frac{h_{k}}{k} x^{2 k}=\frac{1}{4} \ln ^{2}\left(\frac{1+x}{1-x}\right) .
$$


This enables us to evaluate a wide variety of interesting series via specialization, differentiation and integration.

First, setting $x=1 / 2$, we find

$$
\sum_{k=1}^{\infty} \frac{h_{k}}{2^{2 k} k}=\frac{1}{4} \ln ^{2} 3
$$

For $x=\sqrt{2} / 2$,

$$
\sum_{k=1}^{\infty} \frac{h_{k}}{2^{k} k}=\frac{1}{4} \ln ^{2}(3+2 \sqrt{2}) .
$$

Putting $x=(\sqrt{5}-1) / 2=\phi$, the golden ratio, we get

$$
\sum_{k=1}^{\infty} \frac{h_{k}}{k} \phi^{2 k}=\frac{1}{4} \ln ^{2}(2+\sqrt{5}) .
$$

Furthermore, for any $\alpha \geq 2$, putting $x=(\sqrt{5}+1) / 2 \alpha$ and $x=(\sqrt{5}-1) / 2 \alpha$ in (3) respectively, we get

$$
\sum_{k=1}^{\infty} \frac{h_{k}}{\alpha^{2 k} k}\left(\frac{\sqrt{5}+1}{2}\right)^{2 k}=\frac{1}{4} \ln ^{2}\left(\frac{(2 \alpha+1)+\sqrt{5}}{(2 \alpha-1)-\sqrt{5}}\right)
$$

and

$$
\sum_{k=1}^{\infty} \frac{h_{k}}{\alpha^{2 k} k}\left(\frac{\sqrt{5}-1}{2}\right)^{2 k}=\frac{1}{4} \ln ^{2}\left(\frac{(2 \alpha-1)+\sqrt{5}}{(2 \alpha+1)-\sqrt{5}}\right) .
$$

Recalling the Fibonacci numbers which are defined by

$$
F_{1}=1, F_{2}=1, F_{k}=F_{k-1}+F_{k-2} \text { for } k \geq 2
$$

and Binet's formula

$$
F_{k}=\frac{1}{\sqrt{5}}\left(\left(\frac{\sqrt{5}+1}{2}\right)^{k}-\left(\frac{1-\sqrt{5}}{2}\right)^{k}\right),
$$

combining (7) and (8), we find

$$
\sum_{k=1}^{\infty} \frac{h_{k}}{\alpha^{2 k} k} F_{2 k}=\frac{\sqrt{5}}{20} \ln \left(\frac{\alpha^{2}+\alpha-1}{\alpha^{2}-\alpha-1}\right) \ln \left(\frac{\alpha^{2}+\alpha \sqrt{5}+1}{\alpha^{2}-\alpha \sqrt{5}+1}\right) .
$$

In particular, for $\alpha=2$

$$
\sum_{k=1}^{\infty} \frac{h_{k}}{2^{2 k} k} F_{2 k}=\frac{\sqrt{5}}{4} \ln 5 \ln (9+4 \sqrt{5}) .
$$


Another step along this path is to change variables. Setting $x=\cos \theta$ in (3) leads to

$$
\sum_{k=1}^{\infty} \frac{h_{k}}{k} \cos ^{2 k} \theta=\ln ^{2}(\cot (x / 2)) .
$$

Integrating both sides from 0 to $\pi$, and using

$$
\int_{0}^{\pi} \cos ^{2 k} \theta d \theta=\frac{\pi}{2^{2 k}}\left(\begin{array}{c}
2 k \\
k
\end{array}\right)
$$

and

we find

$$
\int_{0}^{\pi} \ln ^{2}(\cot (x / 2)) d \theta=\frac{\pi^{3}}{4},
$$

$$
\sum_{k=1}^{\infty} \frac{h_{k}}{2^{2 k} k}\left(\begin{array}{c}
2 k \\
k
\end{array}\right)=\frac{\pi^{2}}{4} .
$$

This adds another interesting series to Lehmer's list [6].

Next, for $0<x<1$, differentiating (3), then multiplying both sides by $x$, we obtain

$$
\sum_{k=1}^{\infty} h_{k} x^{2 k}=\frac{x}{2\left(1-x^{2}\right)} \ln \left(\frac{1+x}{1-x}\right) .
$$

Setting $x=1 / 2$, we get

$$
\sum_{k=1}^{\infty} \frac{h_{k}}{2^{2 k}}=\frac{1}{3} \ln 3
$$

For $x=\sqrt{2} / 2$

$$
\sum_{k=1}^{\infty} \frac{h_{k}}{2^{k}}=\frac{\sqrt{2}}{2} \ln (3+2 \sqrt{2}) .
$$

Similar to (10), we have

$$
\sum_{k=1}^{\infty} \frac{h_{k}}{2^{2 k}} F_{2 k}=\frac{\sqrt{5}}{50}(10 \ln (5+2 \sqrt{5})+3 \sqrt{5} \ln 5-5 \ln 5) .
$$

Finally, for $0<x \leq 1$, dividing both sides of (3) by $x$ and integrating from 0 to $x$, we obtain

$$
\sum_{k=1}^{\infty} \frac{h_{k}}{k^{2}} x^{2 k}=\frac{1}{2} \int_{0}^{x} \frac{1}{t} \ln ^{2}\left(\frac{1+t}{1-t}\right) d t
$$

Using the substitution $u=(1-x) /(1+x)$ and integration by parts, we get

$$
\begin{aligned}
\sum_{k=1}^{\infty} \frac{h_{k}}{k^{2}} x^{2 k} & =\int_{(1-x) /(1+x)}^{1} \frac{\ln ^{2} u}{1-u^{2}} d u \\
& =\frac{1}{2} \ln x \ln ^{2}\left(\frac{1-x}{1+x}\right)+\int_{(1-x) /(1+x)}^{1} \frac{\ln u}{u} \ln \left(\frac{1-u}{1+u}\right) d u
\end{aligned}
$$


In view of (2), we have

$$
\int_{(1-x) /(1+x)}^{1} \frac{\ln u}{u} \ln \left(\frac{1-u}{1+u}\right) d u=-2 \sum_{k=0}^{\infty} \frac{1}{2 k+1} \int_{(1-x) /(1+x)}^{1} u^{2 k} \ln u d u
$$

Since

$$
\int u^{2 k} \ln u d u=\frac{1}{2 k+1} u^{2 k+1} \ln u-\frac{1}{(2 k+1)^{2}} u^{2 k+1}+C,
$$

we find

$$
\begin{aligned}
\sum_{k=1}^{\infty} \frac{h_{k}}{k^{2}} x^{2 k}= & \frac{1}{2} \ln x \ln ^{2}\left(\frac{1-x}{1+x}\right)+2 \ln \left(\frac{1-x}{1+x}\right) \sum_{k=0}^{\infty} \frac{1}{(2 k+1)^{2}}\left(\frac{1-x}{1+x}\right)^{2 k+1} \\
& +2 \sum_{k=0}^{\infty} \frac{1}{(2 k+1)^{3}}-2 \sum_{k=0}^{\infty} \frac{1}{(2 k+1)^{3}}\left(\frac{1-x}{1+x}\right)^{2 k+1} .
\end{aligned}
$$

In terms of the polylogarithm function [5]

$$
L i_{n}(x)=\sum_{k=1}^{\infty} \frac{x^{n}}{k^{n}}
$$

and noting that

$$
\sum_{k=0}^{\infty} \frac{x^{n}}{(2 k+1)^{n}}=\frac{1}{2}\left(L i_{n}(x)-L i_{n}(-x)\right)
$$

and

$$
\sum_{k=0}^{\infty} \frac{1}{(2 k+1)^{3}}=\sum_{k=0}^{\infty} \frac{1}{k^{3}}-\sum_{k=0}^{\infty} \frac{1}{(2 k)^{3}}=\frac{7}{8} \zeta(3)
$$

we finally obtain

$$
\begin{gathered}
\sum_{k=1}^{\infty} \frac{h_{k}}{k^{2}} x^{2 k}=\frac{7}{4} \zeta(3)+\frac{1}{2} \ln x \ln ^{2}\left(\frac{1-x}{1+x}\right) \\
+\ln \left(\frac{1-x}{1+x}\right)\left(L i_{2}\left(\frac{1-x}{1+x}\right)-L i_{2}\left(\frac{x-1}{1+x}\right)\right)-\left(L i_{3}\left(\frac{1-x}{1+x}\right)-L i_{3}\left(\frac{x-1}{1+x}\right)\right) .
\end{gathered}
$$

Setting $x=1$, we get

$$
\sum_{k=1}^{\infty} \frac{h_{k}}{k^{2}}=\frac{7}{4} \zeta(3)
$$

For $x=1 / 3$,

$$
\begin{aligned}
\sum_{k=1}^{\infty} \frac{h_{k}}{3^{2 k} k^{2}} & =\frac{7}{8} \zeta(3)-\frac{1}{2} \ln 3 \ln ^{3} 2 \\
& +\frac{1}{3} \ln ^{3} 2+\ln 2 L i_{2}(-1 / 2)+L i_{3}(-1 / 2)
\end{aligned}
$$


where we have used

$$
\begin{aligned}
\operatorname{Li}_{2}(1 / 2) & =\frac{\pi^{2}}{12}-\frac{1}{2} \ln ^{2} 2 \\
\operatorname{Li}_{3}(1 / 2) & =\frac{7}{8} \zeta(3)+\frac{1}{6} \ln ^{3} 2-\frac{\pi^{2}}{12} \ln 2 .
\end{aligned}
$$

Moreover, noting that

$$
h_{k}=\sum_{i=1}^{k} \int_{0}^{1} x^{2(i-1)} d t=\int_{0}^{1}\left(\sum_{i=1}^{k} x^{2(i-1)}\right) d t=\int_{0}^{1} \frac{1-x^{2 k}}{1-x^{2}} d x
$$

and rewriting (8) as

$$
\sum_{k=1}^{\infty} \frac{h_{k}}{k^{2}}\left(1-x^{2 k}\right)=\frac{1}{2} \int_{x}^{1} \frac{1}{t} \ln ^{2}\left(\frac{1+t}{1-t}\right) d t
$$

we have

$$
\begin{aligned}
\sum_{k=1}^{\infty} \frac{h_{k}^{2}}{k^{2}} & =\sum_{k=1}^{\infty} \frac{h_{k}}{k^{2}} \int_{0}^{1} \frac{1-x^{2 k}}{1-x^{2}} d x \\
& =\frac{1}{2} \int_{0}^{1}\left(\frac{1}{1-x^{2}} \int_{x}^{1} \frac{1}{t} \ln ^{2}\left(\frac{1+t}{1-t}\right) d t\right) d x
\end{aligned}
$$

Exchanging the order of the integration, we get

$$
\begin{aligned}
\sum_{k=1}^{\infty} \frac{h_{k}^{2}}{k^{2}} & =\frac{1}{2} \int_{0}^{1}\left(\frac{1}{t} \ln ^{2}\left(\frac{1+t}{1-t}\right) \int_{0}^{t} \frac{1}{1-x^{2}} d x\right) d t \\
& =\frac{1}{4} \int_{0}^{1} \frac{1}{t} \ln ^{3}\left(\frac{1+t}{1-t}\right) d t
\end{aligned}
$$

Using the substitution $x=(1-t) /(1+t)$ and the well-known fact that

$$
\int_{0}^{1} x^{k} \ln ^{3} x d x=-\frac{6}{(k+1)^{3}}
$$

we find

$$
\begin{gathered}
\sum_{k=1}^{\infty} \frac{h_{k}^{2}}{k^{2}}=-\frac{1}{2} \int_{0}^{1} \frac{\ln ^{3} x}{1-x^{2}} d x \\
=-\frac{1}{2} \sum_{k=0}^{\infty} \int_{0}^{1} x^{2 k} \ln ^{3} x d x=3 \sum_{k=0}^{\infty} \frac{1}{(2 k+1)^{4}}=\frac{45}{16} \zeta(4)
\end{gathered}
$$

Another path out of (3) is to bring in complex variables. Since

$$
\frac{1}{i} \tan ^{-1}(i z)=\tanh ^{-1} z=\frac{1}{2} \ln \left(\frac{1+z}{1-z}\right)
$$


Replacing $x$ by $i x$ in (3), we obtain

$$
\sum_{k=1}^{\infty} \frac{(-1)^{k-1} h_{k}}{k} x^{2 k}=\left(\tan ^{-1} x\right)^{2} .
$$

This series may be evaluated at values such as $x=2-\sqrt{3}, \sqrt{3} / 3,1$ explicitly:

$$
\begin{gathered}
\sum_{k=1}^{\infty} \frac{(-1)^{k-1} h_{k}(2-\sqrt{3})^{2 k}}{k}=\frac{\pi^{2}}{144}=\frac{3}{72} \zeta(2), \\
\sum_{k=1}^{\infty} \frac{(-1)^{k-1} h_{k}}{3^{k} k}=\frac{\pi^{2}}{36}=\frac{1}{6} \zeta(2), \\
\sum_{k=1}^{\infty} \frac{(-1)^{k-1} h_{k}}{k}=\frac{\pi^{2}}{16}=\frac{3}{8} \zeta(2) .
\end{gathered}
$$

Similarly, applying differentiation and integration to (21), we deduce the corresponding formulas

$$
\begin{gathered}
\sum_{k=1}^{\infty}(-1)^{k-1} h_{k} x^{2 k}=\frac{x}{1+x^{2}} \tan ^{-1} x, \\
\sum_{k=1}^{\infty} \frac{(-1)^{k-1} h_{k}}{k^{2}} x^{2 k}=2 \int_{0}^{x} \frac{\left(\tan ^{-1} t\right)^{2}}{t} d t .
\end{gathered}
$$

In particular, we find

$$
\begin{gathered}
\sum_{k=1}^{\infty} \frac{(-1)^{k-1} h_{k}}{3^{k}}=\frac{\sqrt{3}}{24} \pi, \\
\sum_{k=1}^{\infty} \frac{(-1)^{k-1} h_{k}}{k^{2}}=G \pi-\frac{7}{4} \zeta(3),
\end{gathered}
$$

where $G$ is the Catalan's constant which is defined by

$$
G=\sum_{k=0}^{\infty} \frac{(-1)^{k}}{(2 k+1)^{2}}
$$

Finally, following the excellent suggestion of an anonymous referee, recalling that

$$
h_{k}=H_{2 k}-\frac{1}{2} H_{k}
$$

we find from (19)

$$
\sum_{k=1}^{\infty} \frac{1}{k^{2}} H_{2 k}=\sum_{k=1}^{\infty} \frac{1}{k^{2}} h_{k}+\frac{1}{2} \sum_{k=1}^{\infty} \frac{1}{k^{2}} H_{k}=\frac{11}{4} \zeta(3)
$$


Furthermore, in terms of the multiple series [7]

$$
\sum_{i=1}^{\infty} \sum_{j=1}^{\infty} \frac{1}{i j(i+j)}=2 \zeta(3), \quad \sum_{i=1}^{\infty} \sum_{j=1}^{\infty} \frac{(-1)^{i+j}}{i j(i+j)}=\frac{1}{4} \zeta(3)
$$

the difference gives

$$
\sum_{i, j>1, i+j=\text { odd }} \frac{1}{i j(i+j)}=\frac{7}{8} \zeta(3) .
$$

Setting $i+j=2 k+1$ and using partail fractions, we have

$$
\begin{aligned}
\sum_{i, j>1, i+j=\text { odd }} \frac{1}{i j(i+j)} & =\sum_{k=1}^{\infty} \sum_{j=1}^{2 k} \frac{1}{j(2 k+1-j)(2 k+1)} \\
& =\sum_{k=1}^{\infty} \frac{1}{(2 k+1)^{2}} \sum_{j=1}^{2 k}\left(\frac{1}{j}+\frac{1}{2 k+1-j}\right) \\
& =\sum_{k=1}^{\infty} \frac{1}{(2 k+1)^{2}} 2 H_{2 k} .
\end{aligned}
$$

Thus,

$$
\sum_{k=1}^{\infty} \frac{1}{(2 k+1)^{2}} H_{2 k}=\frac{7}{16} \zeta(3)
$$

Subsequently, we have

$$
\begin{gathered}
\sum_{k=1}^{\infty} \frac{1}{(2 k-1)^{2}} H_{2 k}=\sum_{k=1}^{\infty} \frac{1}{(2 k+1)^{2}} H_{2 k}+ \\
\sum_{k=1}^{\infty} \frac{1}{(2 k-1)^{3}}+\sum_{k=1}^{\infty} \frac{1}{2 k(2 k-1)^{2}}=\frac{21}{16} \zeta(3)+\frac{1}{8}\left(\pi^{2}-8 \ln 2\right) .
\end{gathered}
$$

From this and the known result [n]

$$
\sum_{k=1}^{\infty} \frac{1}{(2 k-1)^{2}} H_{k}=\frac{1}{4}\left(\pi^{2}-\pi^{2} \ln 2-8 \ln 2+7 \zeta(3)\right),
$$

we finally get

$$
\sum_{k=1}^{\infty} \frac{1}{(2 k-1)^{2}} h_{k}=\frac{7}{16} \zeta(3)+\frac{3}{4} \zeta(2) \ln 2
$$

\section{Acknowledgments}

The author would like to thank the referee and the editor for their thoughtful comments and suggestions for improving the original version of the manuscript. 


\section{References}

[1] D. Bailey, J. Borwein and R. Girgensohn, Experimental evaluation of Euler sums, Experimental Math., 3 (1994), 17-30.

[2] J. Borwein, D. Bailey and R. Girgensohn, Experimentation in Mathematics, A. K. Peters, 2004.

[3] J. Borwein and D. V. Bradley, Thirty-two Goldbach variations, preprint. Available at http://users.cs.dal.ca/ jborwein/32goldbach.pdf.

[4] J. Borwein and I. J. Zucker and J. Boersma, The evaluation of character Euler double sums, preprint. Available at http://users.cs.dal.ca/ jborwein/bzb7.pdf.

[5] L. Lewin, Polylogarithms and Associated Functions, Elsevier North Holland, New YorkAmsterdam, 1981.

[6] D. H. Lehmer, Interesting series involving the central binomial coefficient, Amer. Math. Monthly, 92 (1985), 449-457.

[7] D. Zagier, Values of zeta functions and their applications, First European Congress of Mathematics, Vol. 2, Paris, Birkhauser, 1994, pp. 497-512.

2000 Mathematics Subject Classification: Primary 40C15; Secondary 40A25, 11M41, 11M06. Keywords: Euler sums, Riemann zeta function, closed forms.

(Concerned with sequences A000045, A000984, A001008, and A005408.)

Received February 9 2006; revised version received April 21 2006. Published in Journal of Integer Sequences, May 182006.

Return to Journal of Integer Sequences home page. 